\title{
Innate immunity, insulin resistance and type 2 diabetes
}

\author{
J. M. Fernández-Real • J. C. Pickup
}

Received: 13 June 2011 / Accepted: 9 September 2011 / Published online: 30 November 2011

(C) Springer-Verlag 2011

\begin{abstract}
In this edition of 'Then and now' the initial studies by J.C. Pickup and colleagues supporting the hypothesis that type 2 diabetes is caused by activated innate immunity, published in Diabetologia in 1997 (40:1286-1292), are discussed. These initial findings led to research that has uncovered links between insulin resistance, obesity, circulating immune markers, immunogenetic susceptibility, macrophage function and chronic infection. Genetic variations leading to the altered production or function of circulating innate immune proteins, cellular pattern recognition receptors and inflammatory cytokines are linked to obesity, insulin resistance and type 2 diabetes. Components of the innate immune system in the muscle, bone, liver and adipose tissue, as well as macrophages, have been revealed to play a role in systemic insulin action. Evolutionary pressures, such as acute infections at the population level (pandemics) and chronic low exposure to environmental products or infec-
\end{abstract}

J. M. Fernández-Real

University Hospital of Girona 'Dr Josep Trueta',

Girona, Spain

J. M. Fernández-Real

CIBER Fisiopatología de la Obesidad y Nutrición

(CB06/03/010), Spain

URL: www.ciberobn.es

J. C. Pickup

Diabetes Research Group, King's College London School

of Medicine, Guy's Hospital,

London, UK

J. M. Fernández-Real ( $\bowtie)$

Department of Diabetes, Endocrinology and Nutrition, Institut d'Investigació Biomédica de Girona IdibGi,

Avinguda de França s/n,

17007 Girona, Spain

e-mail: jmfreal@idibgi.org tious agents, may have contributed to increased susceptibility and to the current increase in the prevalence of insulin resistance and type 2 diabetes.

Keywords Clinical studies · Cytokines - Glucose tolerance Insulin resistance - Inflammation · Innate immunity · Insulin sensitivity · Macrophages · Pathophysiology

Type 2 diabetes

\begin{abstract}
Abbreviations
GWAS Genome-wide association studies miRNA Micro RNA
\end{abstract}

Then: the origins of activated innate immunity as a factor in the pathogenesis of type 2 diabetes

By the mid 1990s, many components of the pathophysiology of type 2 diabetes had been uncovered. One commonly held view was that acquired factors (such as obesity and underactivity), genetic factors, malnutrition in utero, ageing and 'syndrome X' (now called the metabolic syndrome) result in lipolysis and increased circulating NEFA, which in turn cause insulin resistance via the Randle cycle and impaired beta cell function ('lipotoxicity'). It was thought that this leads to hyperglycaemia, which has a further effect in reducing insulin secretion via 'glucose toxicity' [1]. We began our research into the role of innate immunity in type 2 diabetes at this time, with the aim of exploring whether type 2 diabetes, dyslipidaemia and atherosclerosis were maladaptations of normal physiological responses to injury rather than the result of abnormalities in biochemical pathways. In fact, a marker of the acute-phase response (serum sialic acid) was found to be elevated in people with type 2 diabetes but not in people with type 1 diabetes of 
comparable age, diabetes duration and glycaemic control [2]. This led to the hypothesis that type 2 diabetes and the metabolic syndrome are part of an ongoing cytokineinduced inflammatory or acute-phase response. In our early work, we speculated that this was part of the innate immune system's adaptive reaction to threatened homeostasis, and that this might explain many of the clinical and biochemical features of type 2 diabetes.

The study reported in the paper published in Diabetologia in 1997 [2] was designed to test the theory that the adaptive response of the innate immune system is coupled to clinical and biochemical features of type 2 diabetes. Circulating levels of inflammatory biomarkers were compared in three groups of volunteers: healthy controls, people with type 2 diabetes and no features of the metabolic syndrome, and people with type 2 diabetes and many features of the metabolic syndrome. There was a graded increase in the proinflammatory cytokine IL-6 and acute-phase reactants such as C-reactive protein, with the highest levels observed in type 2 diabetic individuals with the metabolic syndrome, intermediate levels in type 2 diabetic individuals without the syndrome, and the lowest levels in controls. In the paper, the notion was introduced that activated innate immunity and the acute-phase response was a mechanism that could explain a number of well-known characteristics of type 2 diabetes, such as dyslipidaemia, insulin resistance, impaired insulin secretion, accelerated atherosclerosis, central obesity and hyperfibrinogenaemia.

\section{Now: our present understanding of innate immunity and type 2 diabetes}

The concept that activation of innate immunity underlies the pathophysiology of type 2 diabetes, insulin resistance and atherosclerosis $[2,3]$ has been upheld by more recent research. The innate immune system is the body's first-line defence against microbial, chemical, physical and psychological injury, consisting of processes to repair damage, avoid or isolate threats and restore homeostasis. In the last few years, allostasis ('stability through change') in carbohydrate metabolism has also emerged as a physiological adaptation to chronic stress through insulin resistance [4], whereby the continued homeostatic response to acute stress occurs at some cumulative cost to the system [4].

In the originally proposed model, type 2 diabetes and the metabolic syndrome are not due to primary alterations in insulin secretion or action, but to maladaptation of the innate immune system to environmental threats. In the short term, innate immunity provides a survival advantage, allowing the organism to recover from injury. When the response is not proportional to the stimulus, or when the threat is ongoing (e.g. continued overnutrition or inactivity), there is disease instead of repair [2, 3]. In recent years, evidence has accumulated indicating that innate immunity is also inextricably linked to insulin resistance (Fig. 1). This link may involve genetic factors or cellular communication between specialised cells and organs which trigger inflammatory pathways (Fig. 2).

\section{Genetic influences in innate immunity and insulin resistance}

It is likely that individuals have a preset, or inherited, level of innate immunity at birth (as the name innate suggests) that determines their lifetime risk of developing diabetes in the face of adverse environmental stimuli. Genome-wide association studies (GWAS) have now identified many genetic variants that contribute to the risk of type 2 diabetes, including more than 20 common polymorphisms [5]. Loci that are clearly involved in inflammatory pathways or macrophage function have not so far been linked to insulin resistance in GWAS. Instead, most of the identified loci appear to influence the regulation of insulin production by the pancreatic beta cells in the setting of insulin resistance. It might be that the common variants that have been identified account for only a minor fraction of the overall heritable risk. However, a genetic association between the innate immune system and insulin resistance is suggested from studies of the main proinflammatory cytokines. A meta-analysis of more than 20,000 individuals revealed that those with the highest transcription rates of genes encoding TNF $\alpha$ and IL- 6 were prone to developing obesity, insulin resistance and type 2 diabetes, while those with an increased constitutive rate of IL6 transcription showed decreased insulin sensitivity and an increased prevalence of type 2 diabetes [6]. Gene polymorphisms occur in other components of innate immunity that come into play prior to activation of the inflammatory cascade, sensing the presence of non-self molecules, e.g. pattern recognition receptors such as the Toll-like receptor 4, mannose-binding lectin, bactericidal and permeabilityincreasing protein and soluble CD14. These polymorphisms have been linked to insulin sensitivity [7].

Systems biology strategies have also proved helpful in elucidating the link between inflammation and insulin resistance. The gene expression data are treated as a quantitative trait and are integrated with phenotypic data and DNA sequence variations. In a recent study, carefully phenotyped human population and gene expression data for 23,720 transcripts in adipose tissue and blood were available and correlated with clinical traits and genetic variations. A core transcriptional network module that in obese humans is enriched for genes related to inflammatory and immune responses was identified in adipose tissue. 
Fig. 1 In the classical vision ('Then'), type 2 diabetes was considered to be due to primary alterations in insulin secretion (the beta cell) or in insulin action in the insulin-sensitive tissues (muscle, liver, adipose tissue). These tissues were thought to be mere components that passively receive the influence of insulin to increase glucose disposal or restrain glucose production. The main action of insulin was to promote energy storage, while the brain (neurons) was an insulin-independent organ. In the new scenario ('Now'), the insulin-sensitive tissues are active players in the modulation of insulin action (see text) The symbol $\leftrightarrow$ expresses how infiltrating macrophages in different tissues (adipose tissue, liver, muscle, and the recently identified participant, bone) create a cross-talk with systemic insulin action

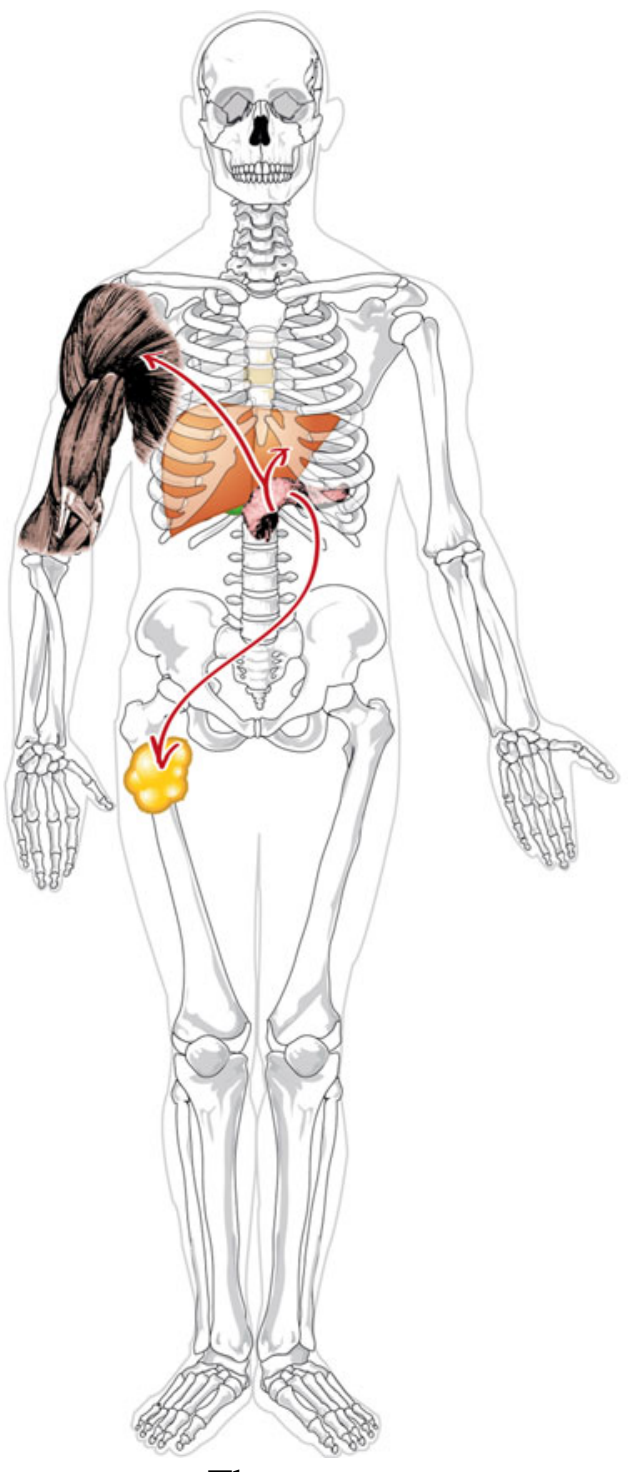

Then

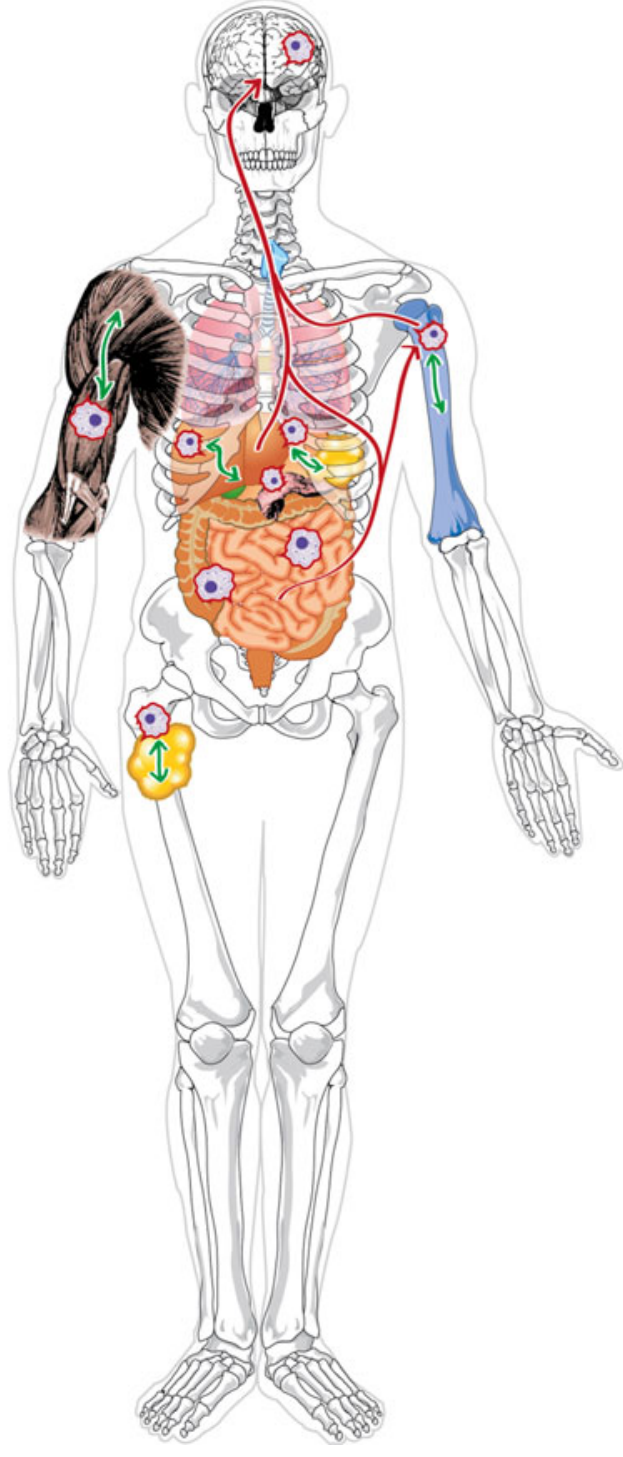

Now
Genome-wide linkage and association mapping analysis revealed a highly significant genetic component to gene

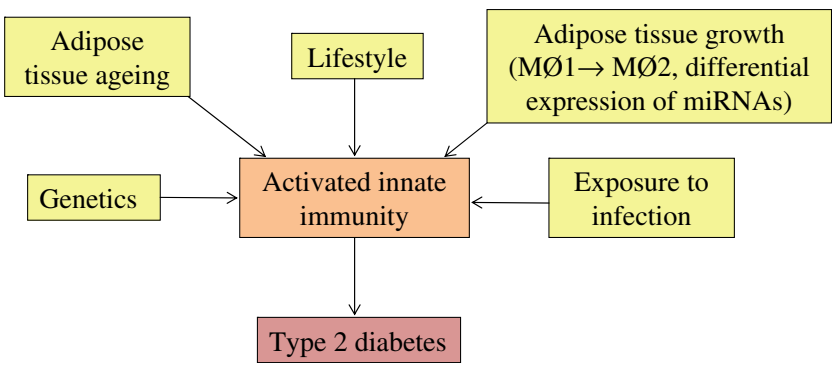

Fig. 2 Different influences on innate immunity thought to lead to the development of type 2 diabetes. It would be useful to study the response to injury as a way of developing new strategies to treat diabetes. For more details, please refer to the main text. МØ, macrophages expression traits. In mice, application of this method resulted in the identification of a macrophage-enriched network, supporting a causal relationship with disease traits associated with the metabolic syndrome [8].

\section{The role of macrophages}

In vertebrates, innate immunity is dependent in large part on myeloid cells, the professional immunocytes that engulf and destroy pathogens. Myeloid cells include mononuclear phagocytes, macrophages derived from blood monocytes, and polymorphonuclear phagocytes. Macrophages are distributed throughout the body, in some cases (e.g. in the heart, brain, lung, and liver) they lie within the parenchyma of major organs as morphologically diverse cells such as the spindle-shaped tissue histiocyte, the flattened Küpffer 
cell of the hepatic sinusoids and the stellate microglial cell of the central nervous system. Macrophages are rarely far away from an invasive organism or the point of chemical or biological insult, and as such are involved in the development of atherosclerosis, insulin resistance and type 2 diabetes.

Of the 100 perigonadal adipose tissue genes that are most strongly correlated with body mass index, 30\% encode proteins that are characteristic of macrophages. Adipose tissue macrophages are responsible for almost all adipose tissue TNF- $\alpha$ and and are the source of significant amounts of inducible nitric oxide synthase and IL-6. Many inflammation- and macrophage-specific genes are dramatically upregulated in the white adipose tissue in mouse models of genetic and high-fat-diet-induced obesity and, in the case of diet-induced obesity, precede a marked increase in circulating insulin levels [7].

Recent research indicates that macrophage types are changed during adipose tissue growth in obesity [9]. Increased numbers of proinflammatory M1 macrophages and decreased anti-inflammatory M2 macrophages were found in the adipose tissue of obese mice. Classical activation leads to the generation of proinflammatory M1 macrophages in response to interferon- $\gamma$, and these play a key role in the phagocytic killing of microbes and the initiation of humoral immunity. These macrophages produce high levels of IL-12, inducible nitric oxide synthase and major histocompatibility complex class II. Alternative activation induces M2 macrophages, which develop in response to IL-4 and IL-13, with anti-inflammatory and angiogenic phenotypes and high levels of anti-inflammatory cytokine IL-10 and arginase production, which depletes the NO donor arginine. Glitazones reduce levels of M1 macrophages and increase levels of M2 macrophages in obese mice. Endoplasmic reticulum stress or hypoxia may contribute to the transition of M2 into M1 macrophages (reviewed in [9]).

Macrophages have been found to be important in the pathophysiology of insulin resistance, in adipose tissue and other tissues. In this sense, macrophages may regulate insulin action in the liver (Küpffer cells), in the muscle (through nucleotide-binding oligomerisation domain [NOD] proteins) and in the bone (osteoclasts modulate the production and activity of osteocalcin, a molecule that potentiates insulin action).

\section{New inroads into the association between the innate immune system and insulin resistance}

As this field expands, there continue to be new layers of complexity in the link between the innate immune system and insulin resistance, which will enrich our understanding of the pathogenesis of type 2 diabetes. Emerging evidence suggests that multiple biological systems and organs, as well as ageing and the microbiota, may trigger the acute-phase response and contribute to the development of type 2 diabetes.

Specific components of innate immunity More than a dozen components of innate immunity have now been linked to metabolic disease [7, 10]. One of the most ancient systems, the complement system, has been recently characterised as a possible modulator of systemic inflammatory activity, not only in the liver but also in adipose tissue.

Proteomics of adipose tissue Study of the myriad of proteins that differ in visceral compared with the more inert subcutaneous tissue may help to unmask the mechanisms linking visceral adipose tissue to insulin resistance and type 2 diabetes. Interestingly, several of these proteins are $100 \%$ specific to visceral adipose tissue and are produced in cells not previously thought to play a role in its inflammatory activity.

MicroRNAs MicroRNAs (miRNAs) were originally recognised as an ancient immune system present in plants. It is increasingly clear that miRNAs are also expressed in the vertebrate immune system and form an immune system themselves. Several miRNAs that regulate innate and acquired immune system function are differentially expressed in the adipose tissue of obese subjects. The change in expression of dozens of miRNAS occurs during adipocyte differentiation, again suggesting a link between the innate immune system and metabolic disease [11].

Ageing of adipose tissue Diabetes is a recognised cause of accelerating ageing, but the mechanisms linking diabetes and ageing are not well understood. Because there are similarities in metabolic dysregulaton in ageing and obesity, it is likely that these conditions share similar cellular pathways. A recent study indicated that adipose tissue from obese mice showed features of premature ageing [12]. The link between obesity and ageing could be abnormal metabolism of the tumour suppressor protein $\mathrm{p} 53$. Inhibition of $\mathrm{p} 53$ activity in adipose tissue markedly ameliorated these senescencelike changes, decreased the expression of proinflammatory cytokines and improved insulin resistance in mice with type 2 diabetes-like disease. One hypothesis is that oxidative stress leads to DNA damage in adipose tissue, telomere dysfunction and activation of p53, leading to increased proinflammatory cytokines and insulin resistance. Activation of p53 occurs in response to shortened 
telomeres in ageing cells. Interestingly, shorter telomeres have been described in adipose tissue cells from obese individuals, being even shorter in formerly morbidly obese individuals compared with never morbidly obese individuals of a similar age and degree of obesity [13].

Body-environment interactions The body is made up of structures that continuously interact and communicate with the environment. To cope with the continuous challenges from this environment, different barriers of defence have emerged through evolutionary pressures. Epithelial cells in the skin constitute the first barrier of defence. Beneath the skin, the body has built an important second line of defence: the adipose tissue, almost $50 \%$ of which is present in the subcutaneous fat depot. In fact, an increased amount of subcutaneous adipose tissue is associated with a lower risk of developing type 2 diabetes.

Mucosal epithelial cells cover the digestive tract, another surface of interaction with the environment. If pathogens are able to disrupt this mucosal barrier, there is another strong line of defence: visceral adipose tissue. This depot is metabolically highly active, unstable and in close contact with the approximately $1-2 \mathrm{~kg}$ of bacteria (the microbiota, see below) and the virome [14] in the gut. If this barrier is overwhelmed, bacterial products and viruses reach the liver, an important buffer of external threats.

Our body also interacts with the environment through the alveolar space and the epithelial cells of the respiratory tract. Surfactant proteins here are also important members of the defence mechanisms associated with insulin action [7].

The primary goal of chronic inflammation and insulin resistance is most likely to ensure glucose delivery to those tissues that are vital for survival during the fight against pathogens: the brain and the immune system (bone marrow). With continuous challenges from the environment (e.g. increased energy intake, a diet high in saturated fat, lack of exercise) or deficiencies in defence (genetic or acquired deficiencies in innate immune cells and proteins), the consequence is chronic low-grade inflammation, resulting in insulin resistance, potentiating each other, producing a vicious cycle that may result in type 2 diabetes.

Microbiota Human beings can be considered as 'superorganisms' as a result of their close symbiotic associations with the gut microbiota. Superorganism metabolism involves the integration of truly indigenous metabolic processes (coded in the host genome) with those of the microbiome. Recent work has shown that the exact human microbiome composition varies between lean and obese individuals, and the microbiome composition is responsive to dietary modulation aimed at weight reduction [15]. In mice, absorption, storage and metabolism of dietary lipids were specifically modulated by the microbiome. A very recent study has demonstrated that intestinal bacteria (green fluorescent-tagged Escherichia coli) can be translocated by immune cells from the intestine towards the adipose tissue by means of the dendritic cells [16].The induction of type 2 diabetes and obesity by a high-fat diet in rats has been shown to correlate with differences in gut bacterial activities, indicating the importance of the microbiome in host predisposition to diseases. The possible interaction of microbiota with inflammatory activity, leading to insulin resistance and type 2 diabetes, is being characterised.

Insulin resistance resulting from chronic inflammation in response to chronic infection? Further evidence for a link between the innate immune system and insulin sensitivity comes from recent observations on exposure to infection [17]. Apparently healthy men with exposure to common pathogens (herpes virus 1, herpes virus 2, enteroviruses and Chlamydia) and characterised by persistent and chronic infection, showed increased fat mass and insulin resistance, and the greater the exposure to these pathogens, the higher the serum levels of inflammatory markers and the lower the insulin sensitivity.

\section{A model for new therapeutic strategies}

The study of the role of innate immunity has uncovered the importance of response to injury in metabolic disease. Every measure used in type 2 diabetes therapy may be envisioned as a way to improve response to injury, from diet (decreasing injury load) to exercise (a way to train the response to injury by repeated micro-injuries), metformin and DPP-IV inhibitors (with their well-known antiinflammatory effects) or insulin itself (a central regulator of inflammation). This model of response to injury should be tested in the future as a way of developing new strategies for the therapy of metabolic disorders.

\section{Conclusions}

The factors that trigger the acute-phase response and contribute to either the development of, or the features of, established type 2 diabetes remain controversial and need much further research. There may be a role for glucose itself, for dietary fat and the $n-3 / n-6$ fatty acid ratio, as well as for obesity, inactivity, ageing, smoking, psychological stress, fetal/neonatal programming and several other factors, such as those implicated in the recognition of microbial surfaces, external and endogenous ligands and their elimination [7, 18]. The pathophysiology of type 2 
diabetes is not restricted to insulin production or insulin effects on the liver, muscle and adipose tissue. Innate immune metabolic pathways may have evolved in adipose tissue, liver, bone and muscle in parallel with mechanisms involved in the fight against infection and injury. It appears that chronic activation of these innate pathways can lead to type 2 diabetes and the conditions that occur in parallel with it, such as atherosclerosis and depression. An understanding of all these processes will lead to a fuller picture of the pathophysiology of insulin resistance and type 2 diabetes.

Acknowledgement We would like to thank P. Tubert (Laboratori d'Enginyeria de Proteïnes, Department of Biology, Facultat de Ciències, University of Girona, Spain) for his help preparing Figure 1.

Contribution statement Both authors participated in the conception and design of the manuscript, drafting the article and revising it critically for important intellectual content. Both authors approved the version to be published.

Duality of interest The authors declare that there is no duality of interest associated with this manuscript.

\section{References}

1. Yki-Järvinen H, Williams G (1997) Insulin resistance in noninsulin-dependent diabetes. In: Pickup JC, Williams G (eds) Textbook of diabetes, 2nd edn. Blackwell Science, Oxford, pp 20.1-20.14

2. Pickup JC, Mattock MB, Chusney GD, Burt D (1997) NIDDM as a disease of the innate immune system: association of acute phase reactants and interleukin-6 with metabolic syndrome $\mathrm{X}$. Diabetologia 40:1286-1292

3. Fernández-Real JM, Ricart W (1999) Insulin resistance and inflammation in an evolutionary perspective. The contribution of cytokine genotype/phenotype to thriftiness. Diabetologia 42:1367-1374
4. Stumvoll M, Tataranni PA, Stefan N, Vozarova B, Bogardus C (2003) Glucose allostasis. Diabetes 52:903-909

5. Bonnefond A, Froguel P, Vaxillaire M (2010) The emerging genetics of type 2 diabetes. Trends Mol Med 16:407-416

6. Huth C, Held IM, Vollmeert C et al (2006) IL6 gene promoter polymorphisms and type 2 diabetes: joint analysis of individual participants' data from 21 studies. Diabetes 55:2915-2921

7. Fernández-Real JM, Pickup JC (2008) Innate immunity, insulin resistance and type 2 diabetes. Trends Endocrinol Metab 19:10 16

8. Emilsson V, Thorleirsson G, Zhang B et al (2008) Genetics of gene expression and its effect on disease. Nature 452:423-428

9. Olefsky JM, Glass CK (2010) Macrophages, inflammation, and insulin resistance. Annu Rev Physiol 72:219-246

10. Fernández-Real JM, Pérez del Pulgar S, Luche E et al (2011) CD14 modulates inflammation-driven insulin resistance. Diabetes 60:2179-2186

11. Ortega FJ et al (2010) MiRNA expression profile of human subcutaneous adipose and during adipocyte differentiation. PLoS One 5:e9022

12. Minamino T, Orimo M, Shimizu I et al (2009) A crucial role for adipose tissue p53 in the regulation of insulin resistance. Nat Med 15:1082-1087

13. Moreno-Navarrete JM, Ortega F, Sabater M, Ricart W, FernándezReal JM (2010) Telomere length of subcutaneous adipose tissue cells is shorter in obese and formerly obese subjects. Int J Obes (Lond) 34:1345-1348

14. Reyes A, Haynes M, Hanson N et al (2010) Viruses in the faecal microbiota of monozygotic twins and their mothers. Nature 466:334-338

15. Turnbaugh PJ, Ley RE, Mahowald MA, Magrini V, Mardis ER, Gordon JI (2006) An obesity-associated gut microbiome with increased capacity for energy harvest. Nature 444:1027-1031

16. Amar J, Chabo C, Waget A et al (2011) Intestinal mucosal adherence and translocation of commensal bacteria at the early onset of type 2 diabetes: molecular mechanisms and probiotic treatment. EMBO Mol Med 3:559-572

17. Krishnapuram R, Dhurandhar EJ, Dubuisson O et al (2011) Template to improve glycemic control without reducing adiposity or dietary fat. Am J Physiol Endocrinol Metab 300: E779-E789

18. Pickup JC (2004) Inflammation and activated innate immunity in the pathogenesis of type 2 diabetes. Diabetes Care 27:813-823 